INPLASY

PROTOCOL

To cite: Jiang et al. Effects of motor imagery training for lower extremity motor function in patients with stroke. Inplasy protocol 2020110037. doi: 10.37766/inplasy2020.11.0037

Received: 09 November 2020

Published: 09 November 2020

Corresponding author:

Rui Qi

qirui36@126.com

Author Affiliation:

Shanghai University of

Traditional Chinese Medicine

Yueyang Hospital of

Integrated Traditional

Chinese Medicine and

Western Medicine

Support: Project Funds.

Review Stage at time of this submission: Data analysis.

Conflicts of interest:

The authors declare no

conflicts of interest.

\section{Effects of motor imagery training for lower extremity motor function in patients with stroke}

\author{
Jiang, L1; Zhao, L2; Qi, R3; Cong, W4; Li, Z5; Zhang, J6.
}

Review question / Objective: The aim of this review is to evaluate the effect of motor imagery training for lower extremity motor function in patients with stroke.

Condition being studied: Motor dysfunction of lower extremity is one of the most common sequela after stroke. Motor imagery training as an emerging rehabilitation technique has been applied to improve the lower extremity motor function in the clinical. However, there is no a vast amount of sufficient evidence on the efficacy of MIT for lower extremity motor function. The aim of this systematic review is to provide more reliable evidence-based medicine evidence for the efficacy of motor imagery training.

INPLASY registration number: This protocol was registered with the International Platform of Registered Systematic Review and Meta-Analysis Protocols (INPLASY) on 09 November 2020 and was last updated on 09 November 2020 (registration number INPLASY2020110037).

\section{INTRODUCTION}

Review question / Objective: The aim of this review is to evaluate the effect of motor imagery training for lower extremity motor function in patients with stroke.
Condition being studied: Motor dysfunction of lower extremity is one of the most common sequela after stroke. Motor imagery training as an emerging rehabilitation technique has been applied to improve the lower extremity motor function in the clinical. However, there is no 
a vast amount of sufficient evidence on the efficacy of MIT for lower extremity motor function. The aim of this systematic review is to provide more reliable evidence-based medicine evidence for the efficacy of motor imagery training.

\section{METHODS}

Participant or population: Patients with stroke.

Intervention: Motor imagery training combined with conventional rehabilitation therapy.

Comparator: Conventional rehabilitation therapy.

Study designs to be included: Randomized controlled trials.

Eligibility criteria: According to our aims, we designed the following inclusion criteria: We will include adults (over 18 years old) suffering from physical dysfunction after a first or recurrent stroke. We also consider RCTs in which a prior history of motor dysfunction before the stroke diagnosis is not investigated but excluded trials reporting on patients with a history of constipation before the stroke diagnosis. Stroke is defined as 'rapidly developed signs of focal or global disturbance of cerebral function, lasting more than $\mathbf{2 4}$ hours or leading to death with no apparent cause other than that of vascular origin, according to WHO criteria. We will include patients with stroke irrespective of any type (ischaemic or haemorrhagic) or phase (acute, subacute or chronic). Acute and subacute stroke is defined as less than 6 months since onset, and chronic stroke lasts more than 6 months since onset.

Information sources: We will search articles in nine electronic databases including: CNKI, WanFang, VIP, CBM, PubMed, Embase and Cochrane Library, Web of Science, ClinicalTrials databases. We will also check reference lists of all identified relevant studies and reviews carefully. These articles also include the studies on the effect of motor imagery training for lower extremity motor function in patients with stroke. These additional researches obtained from references may help us to capture eligible studies as comprehensively as possible.

Main outcome(s): The primary outcome is FMA-LE (Fugl-Meyer assessment scaleLower extremity).

Additional outcome(s): Secondary outcome measures include FAC (Functional Ambulation Classification), BBS (Berg balance scale).

Data management: (1) EndNote $X 9$ and Excel software will be used to extract data. At the same time, the data will be synthesized and stored in Excel chart. (2)Two researchers (Linhong Jiang, Lijuan Zhao) will independently assess abstracts and titles of studies identified by literature search from the electronic databases. Full texts screening and data extraction will be conducted afterwards independently. Any disagreement will be resolved by discussion until consensus is reached or by consulting a third author (Rui Qi). In this step, we will use EndNote. (3) The following data will be extracted: author, year of publication, interventions of experimental groups and control groups, time point, outcome measures, age of patients, total number of people included in the study, patients' basic information, etc. Two researchers (Linhong Jiang, Lijuan Zhao) will separately extract data. Any disagreement regarding data extraction will be will be resolved by discussion until consensus is reached or by consulting a third author (Rui Qi). In this step, we will use Excel.

Quality assessment / Risk of bias analysis: The Cochrane risk of bias tool will be used to evaluate the risk of bias of the included RCTs by two independent reviewers (Zhaoyuan Li and Weiqin Cong. According to the performance of the included literature in the above evaluation items, two researchers will give judgments like low risk, unclear or high-risk judgments 
one by one, and cross-check after completion, respectively. In case of any disagreement, a discussion will be carried out. If no agreement can be reached between the two researchers, a discussion will be made with the researchers in the third researcher (Rui Qi).

Strategy of data synthesis: In this study, statistical analysis will be conducted by using RevMan 5.3 software. Risk ratio (RR) with $95 \%$ confidence intervals (Cls) will be adopted for intervention effect of dichotomous data. Mean difference (MD) with $95 \% \mathrm{Cl}$ will be for intervention effect of continuous data. When measurement methods or units are inconsistent, the standardized mean difference (SMD) with $95 \% \mathrm{Cl}$ will be used to present the intervention effect.

Subgroup analysis: If the included studies have significant statistical heterogeneity, subgroup analysis will be conducted basing on varied parameters that affect the result parameters. These parameters contain the characteristics of patients (for instance, age, sex, different stage of stroke), the characteristics of interventions (for instance type, frequency) and so on.

Sensibility analysis: To evaluate the reliability of our study results, sensitivity analysis will be used. If there is no significant change in the results after deleting the literature, it indicates that the sensitivity is low and our results are reliable. On the contrary, if there is a big difference or even an opposite conclusion after deleting the literature, it indicates a high sensitivity and a low reliability of this study results. When interpreting the results and drawing conclusions, high sensitivity results indicate that there exist potential bias factors related to the effect of the intervention measures.

Language: English and Chinese.

Country(ies) involved: China.

Keywords: Motor imagery training, lower extremity motor function, stroke, protocol, systematic review, metaanalysis.

Contributions of each author:

Author 1 - Linhong Jiang.

Email: 2297881737@qq.com

Author 2 - Lijuan Zhao.

Author 3 - Rui Qi.

Author 4 - Weiqin Cong.

Author 5 - Zhaoyuan Li.

Author 6 - Jianzhong Zhang. 